\title{
LIE COHOMOLOGY OF REPRESENTATIONS OF NILPOTENT LIE GROUPS AND HOLOMORPHICALLY INDUCED REPRESENTATIONS

\author{
BY
} \\ RICHARD PENNEY
}

\begin{abstract}
Let $U$ be a locally injective, Moore-Wolf square integrable representation of a nilpotent Lie group $N$. Let $(\mathcal{X}, \lambda)$ be a complex, maximal subordinate pair corresponding to $U$ and let $\mathcal{K}_{0}=\operatorname{ker} \lambda \cap \mathcal{X}$. The space $C^{\infty}(U)$ of differentiable vectors for $U$ is an $\mathcal{K}_{0}$ module. In this work we compute the Lie algebra cohomology $H^{P}\left(S_{0}, C^{\infty}(U)\right)$ of this Lie module. We show that the cohomology is zero for all but one value of $p$ and that for this specific value the cohomology is one dimensional. These results, when combined with earlier results of ours, yield the existence and irreducibility of holomorphically induced representations for arbitrary (nonpositive), totally complex polarizations.
\end{abstract}

I. Introduction. Let $\mathcal{H}$ be a connected, simply connected, nilpotent Lie algebra over the complex field. Let $\boldsymbol{M}$ be an $\mathcal{H}$ module over $\mathbf{C}$. By $\Lambda^{p}(\mathcal{H}, M)$ we shall mean the space of $M$ valued, alternating, complex $p$-linear forms on $\mathcal{H}$. For $f \in \Lambda^{p}(\mathcal{H}, M), X \in \mathcal{H}$ we define the "canonical action" of $X$ on $f$ by

$$
(X f)\left(X_{1}, \ldots, X_{p}\right)=X\left(f\left(X_{1}, \ldots, X_{p}\right)\right)-\sum_{1}^{p} f\left(X_{1}, \ldots,\left[X, X_{i}\right], \ldots, X_{p}\right) .
$$

This action defines an $\mathcal{H}$-module structure on $\Lambda^{p}(\mathcal{H}, M)$. For $X \in \mathcal{H}$ we also define a mapping $\delta_{X}: \Lambda^{p}(\mathcal{H}, M) \rightarrow \Lambda^{p-1}(\mathcal{H}, M)$ by

$$
\delta_{X} f\left(X_{1}, \ldots, X_{p-1}\right)=f\left(X, X_{1}, \ldots, X_{p-1}\right) .
$$

We define the usual derivation $\partial: \Lambda^{p}(\mathcal{H}, M) \rightarrow \Lambda^{p+1}(\mathcal{K}, M)$ inductively by the equation

$$
\delta_{X}(\partial f)=X f-\partial\left(\delta_{X} f\right)
$$

for all $X \in \mathcal{H}$. (See Hochschild-Serre [3].) It is easily seen that

$$
\begin{aligned}
\partial f\left(X_{1}, \ldots, X_{p+1}\right)= & \sum(-1)^{i} X_{i}\left(f\left(X_{1}, \ldots, X_{i}, \ldots, X_{p+1}\right)\right) \\
& -\sum_{i<j}(-1)^{i+j} f\left(\left[X_{i}, X_{j}\right], X_{1}, \ldots, X_{i}, \ldots, X_{j}, \ldots, X_{p+1}\right) .
\end{aligned}
$$

The corresponding cohomology groups are denoted $H^{p}(\mathcal{H}, M)$.

In this paper we are interested in a specific case of the above construction. Let $N$ be a connected, simply connected nilpotent Lie group over $\mathbf{R}$ with Lie algebra $\Re$. Let $U$ be an irreducible locally injective representation of $N$ which is square

Received by the editors May 8, 1979.

AMS (MOS) subject classifications (1970). Primary 22E25, 22E30, 22 E45.

(c) 1980 American Mathematical Society 0002-9947/80/0000-0401/\$05.75 
integrable modulo its kernel. Let $C^{\infty}(U)$ be the space of infinitely differentiable vectors for $U$. (See [9].) For $v \in C^{\infty}(U)$ and $X \in \Re$, we set

$$
X v=\partial U(X) v=\left.\frac{d}{d t}\right|_{t=0} U(\exp t X) v .
$$

This defines an $\Re$-module structure on $C^{\infty}(U)$. This $\Re$-module structure will be extended as usual to an action of complexification $\Re_{c}$ of $\Re$ on $C^{\infty}(U)$.

Now let $\lambda \in \Re^{*}$ be a linear functional which corresponds to $U$ under the Kirillov correspondence [4]. We extend $\lambda$ to a complex linear functional (also denoted $\lambda$ ) on $\Re_{c}$. A complex subalgebra $\mathscr{H}$ of $\mathscr{T}_{c}$ is subordinate to $\lambda$ if $\lambda$ is null on $[\mathcal{H}, \mathcal{H}] . \mathcal{H}$ is maximal subordinate if $\mathcal{H}$ has maximal dimension among all such subalgebras. Let $\mathcal{K}_{0}=\mathcal{K} \cap$ ker $\lambda$. $\mathcal{H}_{0}$ is a complex subalgebra of $\mathcal{H}$ which is called the reduced subalgebra. In this work we are interested in computing $H^{p}\left(\mathcal{H}_{0}, C^{\infty}(U)\right)$ for arbitrary maximal subordinate pairs $(\mathcal{H}, \lambda)$ corresponding to $U$.

We shall explain our interest in this subject momentarily. First. however, we need some notation. The form $B_{\lambda}(X, Y)=\lambda([X, Y])$ on $\Re_{c} \times \Re_{c}$ is called the antisymmetric form of the pair $(\mathcal{H}, \lambda)$ while the form $\phi_{\lambda}(X, Y)=-i \lambda([X, \bar{Y}])$ on $\mathcal{H}_{0} \times \mathcal{H}_{0}$ is called the Hermitian form of the pair. The subalgebra $\mathscr{R}_{\lambda}$ of elements of $\Re_{c}$ annihilated by $B_{\lambda}$ is called the radical of the pair. A fundamental result of Moore and Wolf [5] says that a locally injective representation $U$ is square integrable iff $\Re_{\lambda}$ is the center of $\Re_{c}$. Local injectivity also implies that the center is one dimensional. It is easily seen that $\phi_{\lambda}$ is Hermitian symmetric. It follows that there is a basis $X_{1}, \ldots, X_{d}$ of $\mathcal{F}_{0}$ such that $\phi_{\lambda}\left(X_{i}, X_{j}\right)=\varepsilon_{i j}$ where $\varepsilon_{i j}$ is zero if $i \neq j$ and $\varepsilon_{i j} \in\{0,-1,+1\}$ for all $i$. The number $p_{0}$ of positive values of $\varepsilon$ is called the signature of the pair $(\mathcal{H}, \lambda)$ and the number $d-p_{0}=q_{0}$ is called the deficit of the pair. Our main result is the following

THEOREM 1. Let $U$ be a locally injective square integrable representation of $N$ and let $(\mathcal{H}, \lambda)$ be a complex, maximal subordinate pair corresponding to $U$. Then $H^{p}\left(\mathcal{F}_{0}, C^{\infty}(U)\right)=0$ if $p \neq q_{0}$ where $q_{0}$ is the deficit of the pair $(\mathcal{H}, \lambda)$. Furthermore $H^{q_{0}}\left(\mathcal{H}_{0}, C^{\infty}(U)\right)$ is one dimensional and the image of $\Lambda^{q_{0}-1}\left(\mathcal{H}_{0}, C^{\infty}(U)\right)$ in $\Lambda^{q_{0}}\left(\mathcal{F}_{0}, C^{\infty}(U)\right)$ under $\partial$ is closed in the $C^{\infty}(U)$ topology.

The $C^{\infty}(U)$ topology is defined as the weakest topology making all maps $v \rightarrow X_{1} X_{2} \cdots X_{n} v$ continuous from $C^{\infty}(U)$ into the representation space of $U$ where $X_{i} \in \Re$ and $n \in \mathbf{N}$. The corresponding topology on $\Lambda^{p}\left(\mathcal{F}_{0}, C^{\infty}(U)\right)$ is obtained by identifying $\Lambda^{p}\left(\mathcal{F}_{0}, C^{\infty}(U)\right)$ with $\Lambda^{p}\left(\left(\mathcal{F}_{0}\right)^{*}\right) \otimes C^{\infty}(U)$.

Our interest in this theorem is that it implies the existence and irreducibility of holomorphic induction for not necessarily positive polarizations of nilpotent Lie groups. Specifically, suppose $\mathcal{H}$ satisfies, in addition to the above assumptions,

(i) $\mathcal{H}+\overline{\mathcal{H}}=\mathscr{K}_{c}$,

(ii) $\mathscr{H} \cap \overline{\mathcal{H}}=\mathscr{R}_{\lambda}=\mathscr{Z}\left(\mathscr{T}_{c}\right)$.

Such subalgebras are called totally complex. In our previous work [8], we showed that the existence and irreducibility of holomorphic induction for the pair $(\mathcal{H}, \lambda)$ was equivalent to the fact that $H^{p}\left(\mathcal{H}_{0}, \lambda\right)$ is one dimensional when $p$ equals the 
deficit and zero otherwise (Theorems 10 and 2). We refer the reader to [8] for details and applications.

The existence and irreducibility of holomorphic induction has been proven previously only in special cases. Camora [1] and Satake [10] proved it for the Heisenberg group. Moscovici [7] proved it for groups which admit rational forms in the case that $(\mathcal{H}, \lambda)$ satisfies a certain rationality assumption and $\lambda$ is "sufficiently distant" from 0. In our previous work [8], we established the existence and irreducibility in the case that $\mathcal{H}$ is abelian. Moscovici has also shown the vanishing part of our Theorem 1 above in the case that $\lambda$ is "sufficiently distant" from zero [7]. ${ }^{1}$

Several interesting features of Theorem 1 are worth mentioning. First, the theorem does not assume that the subalgebra $\mathcal{H}$ is totally complex. One can in fact use our theorem to prove irreducibility in the case $\mathcal{H}$ is real. This suggests that there should be a way of defining realizations of $U$ corresponding to arbitrary complex maximal subordinate pairs $(\mathcal{H}, \lambda)$. Actually, the elimination of the totally complex assumption seems to be an essential ingredient of the proof of Theorem 1 , as our induction scheme forces us out of the totally complex case.

The second interesting comment concerning Theorem 1 is that its proof is constructive: Given a form $f$ which is homologous to zero we could (given time) explicitly construct a form $f_{0}$ such that $\partial f_{0}=f$.

II. Proofs. Our main tool in the proof of Theorem 1 is the lemma stated below. Let $C^{p}, p \geqslant 0$ be a differential complex of vector spaces over $\mathbf{C}$ and let $\partial: C^{*} \rightarrow C^{*}$ and $\delta: C^{*} \rightarrow C^{*}$ be differentials of degree +1 and -1 respectively. Let $X=\partial \delta+$ $\delta \partial$. It is easily seen that $X$ commutes with $\partial$ and $\delta$ and $X$ is of degree 0 . Let

$$
C_{1}^{p}=\left(\delta C^{p+1}+X C^{p}\right) / X C^{p}=\delta C^{p+1} / \delta C^{p+1} \cap X C^{p} \text {. }
$$

The space $\delta C^{p+1}+X C^{p}$ is $\partial$ invariant since $\partial \delta=X-\delta \partial$ and $\partial$ commutes with $X$. It follows that $\partial$ induces a derivation $\partial_{X}$ of the complex $C_{1}^{p}$.

There is also a dual concept related to the kernel of $X$. Let $C_{0}^{p}=\operatorname{ker} X \cap \operatorname{ker} \delta$.

On $C_{0}^{p}, \delta$ and $\partial$ anticommute so again $\partial$ gives rise to a derivation $\partial_{X}$ of $C_{0}^{p}$.

LEMMA 2. If $X$ is injective on each $C^{p}$ then $\delta$ induces an isomorphism between $H^{p}\left(C^{*}\right)$ and $H^{p-1}\left(C_{1}^{*}\right)$ for $p>0$. Also $H^{0}\left(C^{*}\right)=0$.

If $X$ is surjective on each $C^{p}$ then the injection of $C_{0}^{p}$ in $C^{p}$ induces an isomorphism between $H^{p}\left(C_{0}^{*}\right)$ and $H^{p}\left(C^{*}\right)$ for all $p$.

Proof. We first consider the injective case. The identity $\partial \delta=-\partial \delta+X$ shows that $\delta$ anticommutes with $\partial$ modulo the image of $X$ so $\delta$ induces a chain map $\delta_{X}$ of $C^{p}$ into $C_{1}^{p-1}$. Let the corresponding map on cohomology be $\delta_{X}^{*}$.

To see that $\delta_{X}^{*}$ is injective, suppose $f$ is a $\partial$ closed element of $C^{p}$ and $\delta f$ is a boundary in $C_{1}^{p}$. Then $\delta f=\partial g+X h$ for some $g \in \delta C^{p}$. We claim that $\partial h=f$ so $f$ is a boundary in $C^{p}$. In fact

$$
X \partial h=\partial X h=\partial \delta f=X f-\delta \partial f=X f .
$$

\footnotetext{
${ }^{1}$ AdDEd IN Proof. Recently J. Rosenberg has also proven the general result using some of our results below.
} 
Hence $\partial h=f$ as claimed.

To see that $\delta_{X}^{*}$ is surjective, let $g=\delta g_{1}+X C^{p}$ be a $\partial_{X}$ closed coset of $C_{1}^{p}$. Then $\partial \delta g_{1}=X h$ in $C^{p+1}$. We claim that $h$ is closed in $C^{p+1}$ and $\delta h=g \bmod X C^{p} . h$ is closed because $X \partial h=\partial X h=\partial^{2} \delta g_{1}=0$. Also

$$
X \delta h=\delta X h=\delta \partial \delta g_{1}=(\delta \partial+\partial \delta) \delta g_{1}=X \delta g_{1} .
$$

Hence $\delta h=\delta g_{1}=g \bmod X C^{p}$, as claimed.

If $p=0, X=\delta \partial$; so $X$ injective implies ker $\partial=0$ as claimed.

Now suppose $X$ is surjective. Let $i: C_{0}^{p} \rightarrow C^{p}$ be the injection. $i$ is a chain map. Let $i^{*}$ be the induced map on cohomology. We first show that $i^{*}$ is surjective. Let $f \in C^{p}, \partial f=0$. Let $h \in C^{p}$ be such that $X h=f$. Then we claim that $f-\partial \delta h=g$ belongs to $C_{0}^{p}$. Clearly $i^{*}$ maps the class of $g$ onto that of $f$. To see that $g$ belongs to $C_{0}^{p}$, we compute

$$
\begin{aligned}
& \delta g=\delta f-\delta \partial \delta g=\delta f-\delta(X-\delta \partial) g=\delta f-\delta f=0 \\
& X g=X f-\partial \delta X h=\partial \delta f-\partial \delta f=0 .
\end{aligned}
$$

To see that $i^{*}$ is injective suppose $g \in C_{0}^{p}$ and $g=\partial f$ for some $f \in C^{p-1}$. We claim $g=\partial h$ for some $h$ in $C_{0}^{p-1}$. To see this, let $h_{0}$ be such that $X h_{0}=f$ and set $h=f-\partial \delta h_{0}$. Then $g=\partial h$. To see that $h \in C_{0}^{p-1}$, we again compute

$$
\begin{aligned}
& \delta h=\delta f-\delta \partial \delta h_{0}=\delta f-\delta X h_{0}=\delta f-\delta f=0, \\
& X h=X f-\delta \partial X h_{0}=X f-\delta \partial f=\delta \partial f-\delta \partial f=0 .
\end{aligned}
$$

This proves $g$ is null in $H^{p}\left(C_{0}^{*}\right)$ and hence our lemma is proven.

We shall also require a topological version of the above proposition. Suppose that the $C^{p}$ are locally convex topological vector spaces and that $\delta$ and $\partial$ are both continuous. We endow $H^{p}\left(C^{*}\right), C_{i}^{p}$ and $H^{p}\left(C_{i}^{*}\right), i=0,1$, with their respective quotient (subspace) topologies (which may be non-Hausdorff). The mappings $\delta_{X}^{*}$ and $i^{*}$ are continuous, bijective mappings of vector spaces.

LEMMA 3. If $X$ has a continuous inverse $W$ defined on the closure of the image of $X$, then $\delta_{X}^{*}$ is a topological isomorphism. If $X$ is open, $i^{*}$ is a topological isomorphism.

Proof. From the proof of the above proposition, the inverse of $\delta_{X}^{*}$ is given by mapping cosets of the form $g+X C^{p}$ into the cohomology class of $W \partial g$. This is clearly continuous.

To prove the statement about $i^{*}$, let $X$ be an open mapping. We shall show that $i^{*}$ is open. Let $\mho_{0}$ be an open subset of $H^{P}\left(C_{0}^{*}\right)$. By definition of the quotient topology, $\mathscr{U}_{0}$ is a projection to $H^{p}\left(C_{0}^{*}\right)$ of a set of the form $\mathscr{U} \cap C_{0}^{p} \cap \operatorname{ker} \partial$ where $\mathscr{Q}$ is open in $C^{p} \cdot i^{*} \mathscr{U}_{0}$ will be open iff $\mathscr{U} \cap C_{0}^{p} \cap \operatorname{ker} \partial+\partial C^{p-1}$ is open in ker $\partial$. From the proof of Lemma 2 , if $h$ is any element of $C^{p}$ and $\partial X h=0$, then $X h-\partial \delta h$ belongs to $C_{0}^{p} \cap \operatorname{ker} \partial$. Let $\tilde{Q}=(X-\partial \delta)^{-1} \mathscr{Q}$. Then $\tilde{\mathcal{U}}$ is open so $X \tilde{\mathcal{U}}$ is open in $C^{p}$. Thus $X \tilde{\text { U }} \cap$ ker $\partial$ is open in ker $\partial$. It $X h \in X \tilde{\mathcal{U}} \cap$ ker $\partial$ for some $h \in \tilde{Q}$, then $X h-\partial \delta h \in \mathcal{U} \cap \operatorname{ker} \partial \cap C_{0}^{p}$ so

$$
X \tilde{\text { U }} \cap \text { ker } \partial+\partial C^{p-1} \subset \mathcal{U} \cap \text { ker } \partial \cap C_{0}^{p}+\partial C^{p-1} \text {. }
$$

In fact it is not difficult to see that the " $\subset$ " above is really an equality. This proves the lemma. 
In our applications of the above lemma, $\partial$ will be the derivation of $\Lambda^{p}\left(\mathcal{F}_{0}, C^{\infty}(U)\right)$ and $\delta$ will be the map $\delta_{X} f\left(X_{1} \cdots X_{p-1}\right)=f\left(X, X_{1}, \ldots, X_{p-1}\right)$ for some fixed $X \in \mathcal{K}_{0}$. The inductive definition of $\partial$ shows that $\partial \delta_{X}+\delta_{X} \partial$ is simply the usual action of $X$ on $\Lambda^{p}\left(\mathcal{F}_{0}, C^{\infty}(U)\right)$. Hence $\partial \delta_{X}+\delta_{X} \partial=X$. The crux of our proof of Theorem 1 will be to find appropriate $X$ 's and to describe $C_{0}^{p}$ or $C_{1}^{p}$ as the case may be.

We now proceed to the proof of Theorem 1.

Proof. Let $\mathscr{Z}(\Re)$ denote the center of $N . \mathscr{Z}(\Re)$ is one dimensional by virtue of the local injectivity of $U$. There is an element $Y \in \Re$ such that $Y \notin \mathscr{Z}(\Re)$ but $[\Re, Y] \subset \mathscr{Z}(\Re)$. We may pick $Y$ so that $\lambda(Y)=0$. Let $\Re_{1}$ be the centralizer of $Y$ in $\Re$. $\Re_{1}$ is the kernel of ad $Y . \Re_{1}$ has codimension one in $\Re$ as ad $Y$ has a one-dimensional image. Hence $\varkappa_{1}$ is an ideal in $\Re$. Let $N_{1}$ be the corresponding subgroup of $N$. Let $\lambda_{1}=\lambda \mid \Re_{1}$ and let $U_{1}$ be the irreducible representation corresponding to $\lambda_{1}$ under the Kirillov correspondence. Let $U_{1}$ be realized in the Hilbert space $\mathcal{G}_{1}$. It is a well-known consequence of Kirillov theory that $U$ is equivalent with the representation induced from $N_{1}$ by $U_{1}$. Let $X_{0} \in \Re$ be complementary to $\Re_{1}, X_{0}$ may be chosen so $\lambda\left(\left[X_{0}, Y\right]\right)=1$. Let $Z=\left[X_{0}, Y\right]$. We use $X_{0}$ as usual to realize $U$ in $L^{2}\left(R, \mathscr{K}_{1}\right)$ by restriction of functions to the one-parameter subgroup defined by $X_{0}$. The action of $U$ on $L^{2}\left(R, \mathscr{K}_{1}\right)$ is described by the equations

$$
\begin{aligned}
& \left(U\left(\exp t X_{0}\right) f\right)(s)=f(s+t), \quad t \in \mathbf{R}, \\
& (U(x) f)(s)=U_{1}\left(x^{s}\right)(f(s)), \quad x \in N_{1},
\end{aligned}
$$

where $x^{s}=\left(\exp -s X_{0}\right) x\left(\exp s X_{0}\right)$.

We shall require a description of the space $C^{\infty}(U)$ relative to the above realization.

LEMMA 4. A function $f$ in $L^{2}\left(\mathbf{R}, \mathcal{K}_{1}\right)$ is in $C^{\infty}(U)$ iff $f$ is a $C^{\infty}\left(U_{1}\right)$-valued Schwartz map of $\mathbf{R}$. That is to say, $f$ is $C^{\infty}$ as a mapping of $\mathbf{R}$ into $C^{\infty}\left(U_{1}\right)$ and for any polynomial function $p$ and integer $n$, the function $p(d / d t)^{n} f$ is bounded as a map of $\mathbf{R}$ into $C^{\infty}\left(U_{1}\right)$.

Proof. This is not difficult to prove from a few basic facts about $C^{\infty}$ vectors. However, it is somewhat quicker to use results of Corwin-Greenleaf-Penney [2]. Specifically, there is a connected subgroup $H_{1}$ of $N_{1}$ and a character $\chi$ of $H_{1}$ such that $U_{1}$ is the representation of $N_{1}$ induced by $\chi$ and $U$ is the representation of $N$ induced by $\chi$. Let $T_{1}$ be a vector complement to $\log H_{1}$ in $\Re_{1}$ and let $T_{1}=\exp \mathcal{T}_{1}$. We use $T_{1}$ to define a realization of $U_{1}$ in $L^{2}\left(\sigma_{1}\right)$ by restriction of functions to the cross-section $T_{1}$ and composition with the exponential map. Similarly we define a realization of $U$ in $L^{2}\left(\mathcal{T}_{1} \times \mathbf{R}\right)$ by means of the mapping $(t, s) \rightarrow \exp t \exp s X_{0}$ for $t \in \mathcal{T}_{1}, s \in \mathbf{R}$. The results of [2] imply that in these realizations $C^{\infty}\left(U_{1}\right)$ is the Schwartz space $\delta\left(\mathcal{T}_{1}\right)$ of rapidly decreasing $C^{\infty}$ functions on $\mathcal{T}_{1}$ while $C^{\infty}(U)$ is the Schwartz space on $\mathscr{T}_{1} \times \mathbf{R}$. Our lemma follows easily from the fact that $\mathcal{S}\left(\mathcal{T}_{1} \times \mathbf{R}\right)$ can be identified with the space of Schwartz maps of $\mathbf{R}$ into $\mathcal{S}\left(\mathcal{T}_{1}\right)$. Q.E.D. 
Our argument now splits into three cases depending on whether $Y \in \mathcal{H}$ or $Y \in \mathscr{H}+\overline{\mathcal{H}}$ or $Y \notin \mathcal{H}+\overline{\mathcal{H}}$. First, however, let us make some preliminary observations concerning $U_{1} . Y$ is central in $\Re_{1}$ so $U_{1}$ is scalar on $Y$ and the value of $U_{1}$ on $\exp s Y$ is $e^{2 \pi_{i} \lambda_{1}(s Y)} I=I$. Hence $\exp s Y$ belongs to the kernel of $U_{1}$. We shall let / $Y$ be a generic symbol for concepts on $\Re_{1}$ or $N_{1}$ reduced modulo $Y$. For example $U_{1} / Y$ is the representation of $N_{1} / \tilde{Y}$ where $\tilde{Y}$ is the subgroup $\{\exp s Y \mid s \in$ $\mathbf{R}\}$. We note that $U_{1} / Y$ is a locally injective square integrable representation which corresponds to $\lambda_{1} / Y$. In fact $\varkappa_{1}$ is the orthogonal space of $Y+\mathscr{Z}(\mathcal{R})$ relative to $B_{\lambda_{1}}$ so $\operatorname{span}_{\mathbf{C}} Y+\mathscr{Z}(\Re)$ is the radical of $B_{\lambda_{1}}$. Hence $\mathscr{Z}(\Re) / Y$ is the radical of $B_{\lambda_{1} / Y}$. The radical always contains the center so $\mathscr{Z}(\mathcal{T}) / Y$ is the center of $\mathcal{T}_{1} / Y$. Hence $U_{1} / Y$ is square integrable modulo its kernel by the Moore-Wolf Theorem [5]. $U_{1} / Y$ is nontrivial on $\mathscr{Z}(\mathscr{T}) / Y$ so $U_{1} / Y$ is locally injective. We are now ready to deal with Case I.

Case I. $Y \in \mathcal{H}$. In this case $\lambda([Y, \mathcal{H}])=0$ so $\mathcal{H} \subset\left(\mathscr{T}_{1}\right)_{C}$, Case I will follow by induction from the following lemma.

Lemma $4^{\prime}$. In Case I, $H^{0}\left(\mathcal{F}_{0}, C^{\infty}(U)\right)=0$ and $H^{p}\left(\mathcal{F}_{0}, C^{\infty}(U)\right)$ is topologically isomorphic with $H^{p-1}\left(\mathcal{H}_{0} / Y, C^{\infty}\left(U_{1} / Y\right)\right)$.

Proof. It is easily computed that $\partial U(Y)$ is the mapping of $C^{\infty}(U)$ defined by $\partial U(Y) f(s)=i s f(s)$. Since $Y$ centralizes $\mathcal{K}_{0}$, the canonical action of $Y$ on forms $f$ in $\Lambda^{p}\left(\mathcal{H}_{0}, C^{\infty}(U)\right)$ is just $f \rightarrow \partial U(Y) f$. It follows that Lemma 2 applies with $\delta=\delta_{Y}$ and $X=Y$. The image of $Y$ in $\Lambda^{p}\left(\mathcal{F}_{0}, C^{\infty}(U)\right)$ is the set of forms $s \rightarrow f(s)$ which are zero at $s=0$. It follows that the mapping $f \rightarrow f(0)$ defines an isomorphism of $\Lambda^{p}\left(\mathcal{H}_{0}, C^{\infty}(U)\right) / Y \Lambda^{p}\left(\mathcal{K}_{0}, C^{\infty}(U)\right)$ with $\Lambda^{p}\left(\mathcal{H}_{0}, C^{\infty}\left(U_{1}\right)\right)$. The image in $\Lambda^{p-1}\left(\mathcal{H}_{0}, C^{\infty}(U)\right)$ of $\Lambda^{p}\left(\mathcal{F}_{0} C^{\infty}(U)\right)$ under $\delta_{Y}$ is the space of forms which are zero whenever any argument belongs to the span over $\mathbf{C}$ of $Y$. This in turn is isomorphic with $\Lambda^{p-1}\left(\mathcal{H}_{0} / Y, C^{\infty}(U)\right)$. It follows that the space $C_{1}^{p-1}$ of Lemma 2 is

$$
\delta C^{p} / X C^{p-1} \cap \delta C^{p}=\Lambda^{p-1}\left(\mathcal{H}_{0} / Y, C^{\infty}\left(U_{1}\right)\right) .
$$

Lemma 2 implies the algebraic isomorphism of the spaces in question. The image of $\partial U(Y)$ is clearly closed and multiplication by $(i s)^{-1}$ is continuous on the image of $\partial U(Y)$ by the closed graph theorem. Thus Lemma 3 also applies, proving Lemma 4.

The signature of $\phi_{\lambda_{1} / Y}$ is the same as that of $\phi_{\lambda_{1}}$ while the dimension of $\mathcal{H}_{0} / Y$ decreases by one. Hence the deficit decreases by one. Also the deficit of $\left(\mathcal{F}_{0}, \lambda\right)$ cannot be zero because $Y$ is in the radical of $\phi_{\lambda}$. Therefore the validity of Theorem 1 for $\left(\mathcal{H}_{0} / Y, \lambda_{1} / Y\right)$ implies Theorem 1 for $\left(\mathcal{H}_{0}, \lambda_{1}\right)$ and Case I follows by induction.

Case II. $Y \in \mathcal{H}+\overline{\mathcal{H}}$ and $Y \notin \mathcal{H}$. In this case $\mathcal{H} \not\left(\mathscr{T}_{1}\right)_{c}$ since $Y$ cannot centralize $\mathcal{H}$ by the maximality of $\mathcal{H}$. Let $\mathcal{K}_{1}=\mathcal{K}_{0} \cap\left(\mathscr{K}_{1}\right)_{c}$ and let $\mathcal{K}_{2}=$ $\operatorname{span}_{\mathbf{c}} Y+\mathcal{K}_{1}$.

Case II splits into two subcases.

Subcase II.A. $Y \in \mathcal{H}_{1}+\overline{\mathscr{K}}_{1}$. In this case there is a real $V \in \Re_{1}$ such that $Y+i V \in \mathcal{H}_{1}$. 
Lemma 5. $\partial U(Y+i V)$ is injective on $C^{\infty}(U)$.

Proof. Suppose $\partial U(Y+i V) f=0$. Then $i\left(s+\partial U_{1}\left(V^{s}\right)\right) f(s)=0$ for all $s$. In particular $f(s)$ is an eigenvector for $\partial U_{1}\left(V^{s}\right)$ of eigenvalue $-s$. However $\partial U_{1}\left(V^{s}\right)$ is skew-symmetric so $f(s)=0$ unless $s=0$. The continuity of $f$ then implies $f \equiv 0$. This proves the lemma.

We cannot conclude directly from Lemma 5 that Lemma 2 is applicable as $W=Y+i V$ need not centralize $\mathcal{K}_{0}$. However, for $f \in \Lambda^{p}\left(\mathcal{K}, C^{\infty}(U)\right)$ and $W \in$ $\mathcal{H}$ let

$$
W^{*} f\left(X_{1}, \ldots, X_{p}\right)=\sum_{i} f\left(X_{1}, \ldots,\left[W, X_{i}\right], \ldots, X_{p}\right)
$$

so $W f=\partial U(W) f-W^{*} f$. It is clear that $\partial U(W)$ and the mapping $W^{*}: f \rightarrow W^{*} f$ commute. Furthermore $W^{*}$ is nilpotent on $\Lambda^{p}\left(\mathcal{F}_{0}, C^{\infty}(U)\right)$.

LEMMA 6. If $\partial U(W)$ is injective on $C^{\infty}(U)$ then $f \rightarrow W f$ is injective on $\Lambda^{P}\left(\mathcal{H}_{0}, C^{\infty}(U)\right)$.

Proof. If $W f=0$, then $\partial U(W) f=W^{*} f$. By iteration $\partial U(W)^{n} f=\left(W^{*}\right)^{n} f=0$ if $n$ is sufficiently large. Hence $f=0$, proving the lemma.

Thus Lemma 2 is applicable with $\delta=\delta_{W}$ and $X$ given by the action of $W=Y+i V$ on $\Lambda^{p}\left(\mathcal{H}_{0}, C^{\infty}(U)\right)$. The main difficulty in our treatment of this subcase will be the identification of the image of $W$.

To aid in this identification we introduce a new $\varkappa_{c}$ module. As we shall also use this construction later, we shall be slightly more general than our current needs dictate. Let $X_{1} \in \Re_{c} \sim\left(\Re_{1}\right)_{c}$. We define a mapping $D_{X_{1}}$ of $C^{\infty}(U)$ into the product space $\Pi_{n=1}^{\infty} C^{\infty}\left(U_{1}\right)$ by $D_{X_{1}} f=\left(f_{0}, f_{1}, \ldots\right)$ where $f_{i}=\partial U\left(X_{1}\right)^{n} f(0) / n !$.

The image of $D_{X_{1}}$ is denoted $M_{X_{1}}$ and is called the evaluation module relative to $X_{1}$. We define an action of $\Re_{c}$ on $M_{X_{1}}$ so that $D_{X_{1}}$ is a Lie module homomorphism as follows. For $K \in\left(\Re_{1}\right)_{c}$, let $K_{n}=\left(\operatorname{ad} X_{1}\right)^{n}(K) / n !$. For $f=\left(f_{0}, f_{1}, \ldots\right) \in M_{X_{1}}$ we set $K f=\left(g_{0}, g_{1}, \ldots\right)$ where $g_{n}=\sum_{i+j=n} \partial U\left(K_{i}\right) f_{j}$. We also define $X_{1} f=$ $\left(f_{1}, 2 f_{2}, 3 f_{3}, \ldots\right)$.

The action is extended to the rest of $\pi_{c}$ by linearity.

LEMMA 7. $M_{X_{1}}$ is an $\Re_{c}$ module and $D_{X_{1}}$ is an $\mathfrak{x}_{c}$ module homomorphism. The isomorphism class of $M_{X_{1}}$ is independent of $X_{1}$, and as a vector space $M_{X_{1}}=$ $\prod_{n=0}^{\infty} C^{\infty}\left(U_{1}\right)$.

Proof. The following identity in the complex enveloping algebra $\mathfrak{u}_{c}(\Re)$ of $\Re$ is well known.

$$
X_{1}^{n} K=\sum_{i+j=n}\left(\begin{array}{l}
n \\
j
\end{array}\right)\left(\operatorname{ad} X_{1}\right)^{j}(K) X_{1}^{i}
$$

This implies that

$$
\left(X_{1}^{n} / n !\right) K=\sum_{i+j=n} K_{j}\left(X_{1}^{i} / i !\right)
$$


Hence $D_{X_{1}} K f=K D_{X_{1}} f$. The property $X_{1} D_{X_{1}}=D_{X_{1}} X_{1}$ is clear. The Lie-module property of $M_{X_{1}}$ follows easily from the surjectivity of $D_{X_{1}}$ and the above comments.

To prove the isomorphism of the various modules, assume that $X_{2}$ is another element of $\Re_{c} \sim\left(\Re_{1}\right)_{c}$. From the Poincaré-Birkhoff-Whitt Theorem every element $X$ of $\mathfrak{U}(\mathscr{U})$ is expressible uniquely in the form

$$
X=\sum_{i=0}^{k}\left(C_{i}(X) X_{1}^{i}\right) / i !
$$

where $k$ is some integer depending on $X$ and $C_{i}(X)$ belongs to $\mathfrak{u}_{c}\left(\Re_{1}\right)$. In particular we may write

$$
X_{2}^{n} / n !=\sum_{i=0}^{k(n)}\left(C_{n, i} X_{1}^{i}\right) / i !
$$

Let $C: M_{X_{1}} \rightarrow M_{X_{2}}$ be the mapping $C:\left(f_{0}, f_{1}, \ldots\right) \rightarrow\left(g_{0}, g_{1}, \ldots\right)$ where

$$
g_{n}=\sum_{i=0}^{k(n)} \partial U_{1}\left(C_{n, i}\right) f_{i}
$$

(Here we have extended $\partial U_{1}$ to $\mathfrak{U}_{c}\left(\Re_{1}\right)$ as usual.)

Sublemma. $C D_{X_{1}}=D_{X_{2}} C$.

Proof. Let $f \in C^{\infty}(U)$ and let $f_{i}=\left(\partial U\left(X_{1}\right)^{i} f\right)(0) / i$ !. Then

$$
\left(\partial U\left(C_{n, i} X_{1}^{i}\right) f\right)(0)=\partial U_{1}\left(C_{n, i}\right)\left(\partial U\left(X_{1}\right)^{i} f(0)\right) .
$$

Hence, if $g_{n}$ is as above,

$$
g_{n}=\sum_{i=0}^{k(n)}\left(\partial U\left(C_{n, i} X_{1}^{i}\right) f\right)(0) / i !=\partial U\left(X_{2}^{n}\right) f(0) / n ! .
$$

This proves the sublemma.

We may also define an inverse $\tilde{C}$ to $C$, for, by the same reasoning,

$$
X_{1}^{n} / n !=\sum_{i=0}^{l(n)} \tilde{C}_{n, i} X_{2}^{i} / i !
$$

with $\tilde{C}_{n, i} \in \mathfrak{U}_{c}\left(\Re_{1}\right)$. Hence $\tilde{C}_{n, i}$ gives rise to a mapping $\tilde{C}$ of $M_{X_{2}}$ into $M_{X_{1}}$. The mappings $C$ and $\tilde{C}$ are inverse to each other. In fact

$$
X_{2}^{n} / n !=\sum_{i=0}^{k(n)} C_{n, i} X_{1}^{n} / i !=\sum_{i=0}^{k(n)} \sum_{j=0}^{l(i)} C_{n, i} \tilde{C}_{i, j} X_{2}^{j} / j ! .
$$

Thus

$$
\sum_{i=0}^{k(n)} C_{n, i} \tilde{C}_{i, j}=\delta_{j, n}
$$

showing the inverse property. This proves the isomorphism of the modules.

To prove that $D_{X_{1}}$ maps $C^{\infty}(U)$ onto $\Pi_{n=0}^{\infty} C^{\infty}\left(U_{1}\right)$ for all $X_{1}$, we claim that it suffices to prove this for one single $X_{1}$. In fact, the isomorphism $C$ between $M_{X_{1}}$ and $M_{X_{2}}$ is definable on all of $\prod_{n=0}^{\infty} C^{\infty}\left(U_{1}\right)$ by the same formula. The mapping $C$ 
is still inverse to $C$ so if $D_{X_{1}}$ maps onto the product space for a single $X_{1}$, then it will map onto for all $X_{1}$. We choose $X_{1}=X_{0}$, the (real) element of $\Re$ complementary to $\pi_{1}$ chosen above.

To prove our lemma, let $f=\left(f_{0}, f_{1}, \ldots\right)$ belong to $M_{X_{0}}$. We need to show that there is a function $g \in C^{\infty}\left(U_{1}\right)$ such that

$$
f_{i}=\partial U\left(X_{0}\right)^{n} g(0) / n !=(d / d t)^{n} g(0) / n ! .
$$

Let $\psi: \mathbf{R} \rightarrow \mathbf{R}$ be a $C^{\infty}$ function which is supported in the interval $[-1,1]$ and which is one on a neighborhood of 0 in $\mathbf{R}$. Let $h_{n}$ be the function $h_{n}(t)=t^{n} \psi(t) f_{n}$. Let $\lambda_{n}$ be a sequence of positive numbers and consider the functions

$$
g_{n}(t)=\lambda_{n}^{-n} h_{n}\left(\lambda_{n} t\right)=t^{n} \psi\left(\lambda_{n} t\right) f_{n}
$$

Let $\left\{X_{0}, Y_{1}, Y_{2}, \ldots, Y_{k}\right\}$ be a basis for $\Re$ with $Y_{i} \in \Re_{1}$ and $X_{0}$ as above. It is possible to choose $\lambda_{n}$ large enough so that

$$
\left\|\partial U\left(X_{0}^{n_{0}} Y_{1}^{n_{1}} Y_{2}^{n_{2}} \cdots Y_{k}^{n_{k}}\right) g_{n}\right\|<2^{-n}
$$

whenever $\sum n_{i}<n-2$. This is because

$$
X_{0}^{m} g_{n}=(d / d t)^{m} g_{n}=\lambda_{n}^{m-n}\left(d^{m} / d t^{m}\right) h_{n}\left(\lambda_{n} t\right) .
$$

Then $g=\sum_{n=0}^{\infty} g_{n}$ converges in $C^{\infty}(U)$. It is easily seen that

$$
\partial U\left(X_{0}\right)^{n} g(0) / n !=f_{n} \text {. }
$$

This proves the lemma.

The relevance of the above construction to our current problem is the following lemma. Let $X_{1} \in \Re_{c} \sim\left(\Re_{1}\right)_{c}$.

LEMMA 8. Let $f \in C^{\infty}(U)$; then $f$ is in the image of $\partial U(W)$ iff $D_{X_{1}} f$ is in the image of $W$ in $M_{X_{1}}$. Furthermore $W$ is injective on $M_{X_{1}}$.

Proof. From Lemma 7 it suffices to assume $X_{1}=X_{0}$. It is clear that if $f$ is in the image of $\partial U(W)$ then $D_{X_{0}} f$ is in the image of $W$.

Conversely, suppose $D_{X_{0}} f=W \tilde{g}$. We need to show that $f$ is in the image of $W$. From Lemma 7, there is a $g \in C^{\infty}(U)$ such that $\tilde{g}=D_{X_{0}} g$. Then $D_{X_{0}}(f-W g)=$ 0 so it suffices to consider the case $D_{X_{0}} f=0$.

Now, recall that $W=Y+i V$.

$$
\partial U(Y) f(s)=\partial U_{1}\left(Y^{s}\right) f(s)=i s f(s) .
$$

Hence

$$
\partial U(W) f(s)=i\left(s+\partial U_{1}\left(V^{s}\right)\right) f(s) .
$$

The spectrum of $\partial U_{1}\left(V^{s}\right)$ is purely imaginary so $s+\partial U_{1}\left(V^{s}\right)$ is invertible for $s \neq 0$. The inverse is given by

$$
\begin{aligned}
\left(s+\partial U_{1}\left(V^{s}\right)\right)^{-1} & =\int_{0}^{\infty} \exp -\left(s t+\partial U_{1}\left(t V^{s}\right)\right) d t \\
& =\int_{0}^{\infty} e^{-s t} U_{1}\left(\exp -t V^{s}\right) d t
\end{aligned}
$$


for $s>0$ and

$$
\left(s+\partial U_{1}\left(V^{s}\right)\right)^{-1}=-\int_{0}^{\infty} e^{s t} U_{1}\left(\exp t V^{s}\right) d t
$$

for $s<0$.

These integrals converge in the strong operator topology on the representation space of $U_{1}$. Let $g$ be the mapping of $\mathbf{R}$ into the representation space of $U_{1}$ given by

$$
g(s)=-i\left(s+\partial U_{1}\left(V^{s}\right)\right)^{-1} f(s), \quad s \neq 0, \quad g(0)=0 .
$$

Sublemma. $g \in C^{\infty}(U)$ and $\partial U(W) g=f$.

Proof. From Lemma 4, we need to show that $g$ is a $C^{\infty}$ mapping of $\mathbf{R}$ into $C^{\infty}\left(U_{1}\right)$ and all derivatives of $g$ remain bounded in the $C^{\infty}\left(U_{1}\right)$ topology when multiplied by an arbitrary polynomial.

We begin by showing that $g$ is a polynomially bounded mapping of $\mathbf{R}$ into $C^{\infty}\left(U_{1}\right)$. To see this it suffices to show that $g(s)$ belongs to $C^{\infty}\left(U_{1}\right)$ and $\left\|\partial U_{1}(A) g(s)\right\|$ is polynomially bounded in $s$ for all $A$ in the enveloping algebra $\mathfrak{U}\left(\Re_{1}\right)$. For each $s \in \mathbf{R}$ let $A(s, t)$ denote $e^{t \text { ad } V^{s}}(A)$ where ad $V^{s}$ is extended as usual to the enveloping algebra. Formally, we have the following identity for $s>0$.

$$
\begin{aligned}
\partial U_{1}(A) g(s) & =\int_{0}^{\infty} \partial U_{1}(A) U_{1}\left(\exp -t V^{s}\right) f(s) e^{-s t} d t \\
& =\int_{0}^{\infty} U_{1}\left(\exp -t V^{s}\right) \partial U_{1}(A(s, t)) f(s) e^{-s t} d t
\end{aligned}
$$

The analytic validity of this identity is easily verfied by differentiation under the integral. Note that this implies $g(s) \in C^{\infty}\left(U_{1}\right)$. Now there are fixed elements $A_{1} \cdots A_{k} \in \mathfrak{U}\left(\Re_{1}\right)$ and polynomials $p_{i}(s, t)$ such that

$$
A(s, t)=\sum_{i=1}^{k} p_{i}(s, t) A_{i}
$$

If $p(s, t)$ is any polynomial, then $\int_{0}^{\infty} p(s, t) e^{-s t} d t$ increases at most polynomially in $s$ as $s \rightarrow \infty$. It follows that there are polynomials $q_{i}(s)$ such that

$$
\left\|\partial U\left(A_{1}\right) g(s)\right\| \leqslant \sum_{i=1}^{n} q_{i}(s)\left\|\partial U_{1}\left(A_{i}\right) f(s)\right\| .
$$

The polynomial boundedness of $f$ now is seen to imply the polynomial boundedness of $g(s)$ for large positive $s$. The case of large negative $s$ is proven similarly. The boundedness for all $s$ will follow once we have shown that $g$ is $C^{\infty}$ as a mapping of $\mathbf{R}$ into $C^{\infty}\left(U_{1}\right)$.

The fact that $g$ is $C^{\infty}$ at zero follows from the fact that $\lim _{s \rightarrow 0} f(s) / s^{n}=0$ for all $n$ since $(d / d s)^{n} f(0)=0$. In fact

$$
\lim _{s \rightarrow 0^{+}} g(s) / s^{n}=\lim _{s \rightarrow 0^{+}} \int_{0}^{\infty} s^{2} e^{-s t} U_{1}\left(\exp -t V^{s}\right)\left(f(s) / s^{n+2}\right) d t .
$$

As $s \rightarrow 0^{+}, s^{2} e^{-s t} \rightarrow 0$ in $L^{1}([0, \infty))$ and the rest of the integrand remains bounded so $\lim _{s \rightarrow 0^{+}} g(s) / s^{n}=0$. A similar computation for $s<0$ shows $(d / d s)^{n} g(0)=0$.

To show that $g$ is $C^{\infty}$ at nonzero $s$ we adopt another point of view. Again let 
$s>0$. The function $s \rightarrow U_{1}\left(\exp -t V^{s}\right) f(s)$ is merely

$$
(U(\exp -t V) f)(s)=\left(U\left(\exp s X_{0}\right) U(\exp -t V) f\right)(0) .
$$

Let $\delta: C^{\infty}(U) \rightarrow C^{\infty}\left(U_{1}\right)$ be the map $\delta(f)=f(0)$. For $s>0$ let $T_{s}$ be the map

$$
T_{s} f=\int_{0}^{\infty} e^{-s t} U(\exp -t V) f d t .
$$

Then

$$
g(s)=\delta\left(U\left(\exp s X_{0}\right) T_{s} f\right) .
$$

If we can show that $s \rightarrow U\left(\exp s X_{0}\right) T_{s} f$ is a $C^{\infty}$ mapping of $\mathbf{R}$ into $C^{\infty}(U)$, our claim will follow from the continuity of $\delta$. The fact that $T_{s}$ maps $C^{\infty}(U)$ into $C^{\infty}(U)$ is shown in much the same way that we showed that $g(s) \in C^{\infty}\left(U_{1}\right)$. In fact, if $B \in \mathfrak{U}(\mathcal{U})$, then

$$
\begin{aligned}
\partial U(B) T_{s} f & =\int_{0}^{\infty} e^{-s t} U(\exp -t V) \partial U(B(t)) f \\
& =\sum_{i=1}^{k} \int_{0}^{\infty} e^{-s t} U(\exp -t V) r_{i}(t) \partial U\left(B_{i}\right) f d t
\end{aligned}
$$

where $B(t)=e^{t \text { ad } V} B$ and $r_{i}$ are polynomials. Differentiation with respect to $s$ under the integral is justified if $s>0$ and shows the $C^{\infty}$ nature of $T_{s} f$.

It now follows from the product rule for differentiation that $g$ is $C^{\infty}$ for $s>0$ and

$$
\left(\frac{d}{d s}\right)^{n} g(s)=\delta\left(U\left(\exp s X_{0}\right)\left(\sum_{j=0}^{n}\left(\begin{array}{l}
n \\
j
\end{array}\right) \partial U\left(X_{0}\right)^{n-j} T_{s}^{j} f\right)\right)
$$

where

$$
T_{s}^{j} f=\left(\frac{d}{d s}\right)^{j} T_{s} f=\int_{0}^{\infty}(-1)^{j} t^{j} e^{-s t} U(\exp -t V) f d t .
$$

Bringing the $\partial U\left(X_{0}\right)^{n-j}$ past the integral defining $T_{s}^{j}$ as above we see that

$$
\left(\frac{d}{d s}\right)^{n} g(s)=\sum_{j=1}^{k}\left(\int \sigma_{j}(t) e^{-s t} U(\exp -t V) f^{j} d t\right)(s)
$$

where the $\sigma_{j}$ are certain polynomials and the $f_{j}$ 's are elements of $C^{\infty}(U)$. The proof given above for the polynomial boundedness of $g$ applies to the terms in the above sum as well and shows the polynomial boundedness for $(d / d s)^{n} g(s)$ for $s$ large, positive. Similar arguments apply for negative $s$ and our sublemma is proven.

To finish the lemma, we need only show the injectivity of $W$ on $M_{X_{0}}$. We begin by showing that $\partial U_{1}(W)=i \partial U_{1}(V)$ is injective on $C^{\infty}\left(U_{1}\right)$. (Note that $\exp Y \in$ ker $U_{1}$.) Let $\chi_{0}$ be the character of the center $\mathscr{Z}\left(N_{1}\right)$ of $N_{1}$ defined by

$$
\chi_{0}(x)=\exp i \lambda_{1}(\log x) .
$$

Let $U^{x_{0}}$ be the representation of $N_{1}$ induced by $\chi_{0}$. By results of Moore and Wolf, $U_{1}$ can be realized as a subrepresentation of $U^{x_{0}}$. The operator $\partial U_{1}(V)$ is a left invariant differential operator so $\partial U_{1}(V) f=0$ implies that $f$ is constant on right cosets of $\{\exp t V \mid t \in \mathbf{R}\}$. As $V \notin \mathscr{Z}\left(\Re_{1}\right)=\operatorname{span} Y+\mathscr{L}(N)$, the constancy 
of $f$ along $V$-cosets is inconsistent with the square integrability of $\hat{f}$ unless $f \equiv 0$.

Now suppose $f=\left(f_{0}, f_{1}, \ldots\right) \in M_{X_{0}}$ is annihilated by $V$. Then $\left(V f_{0}=\right.$ $\partial U_{1}(V) f_{0}$ so $f_{0}=0$. But $f_{0}=0$ implies $(V f)_{1}=\partial U_{1}(V) f_{1}$ so $f_{1}=0$. By induction it follows that all $f_{i}=0$ and $V$ is injective, proving the lemma.

It now follows from the proof of Lemma 6 that Lemma 2 applies to the complex $C^{p}=\Lambda^{p}\left(\mathcal{F}_{0}, M\right)$ with $\delta=\delta_{W}$ and $X=W$. Let $X_{1} \in \mathscr{T}_{0} \sim \mathscr{T}_{1}$.

Lemma 9. $H^{p}\left(\mathcal{H C}_{0}, M_{X_{1}}\right)$ and $H^{p}\left(\mathcal{F C}_{0}, C^{\infty}(U)\right)$ are isomorphic. The isomorphism is the map $D_{X_{1}}^{*}$ induced by $D_{X_{1}}$ on homology.

Proof. Let $C^{p}=\Lambda^{p}\left(\mathcal{H}_{0}, C^{\infty}(U)\right)$ and $\tilde{C}^{p}=\Lambda^{p}\left(\mathcal{F C}_{0}, M_{X_{1}}\right)$. Let $\delta$ be the derivation $\delta_{W}$ on $C^{p}$ and $\tilde{\delta}$ be $\delta_{W}$ on $\tilde{C}^{p}$. The previous lemma shows that $D_{X_{1}}$ defines a chain map from $C^{p}$ to $\tilde{C}^{p}$ such that $f$ is in the image of $W=X$ in $C^{p}$ iff $f$ is in the image of $W$ in $\tilde{C}^{p}$. It follows that $D_{X_{1}}$ induces a chain isomorphism of the complexes $C_{1}^{p}$ and $\tilde{C}_{1}^{p}$. Lemma 2 , applied to $C_{1}^{p}$ and $\tilde{C}_{1}^{p}$, now shows that $H^{p}\left(\mathscr{C}_{0}, C^{\infty}(U)\right)$ and $H^{p}\left(\mathcal{C}_{0}, M_{X_{1}}\right)$ are both isomorphic to $H^{p-1}\left(C_{1}^{*}\right)$ for $p>0$ and to zero for $p=0$.

We are not yet finished as we still must show that the isomorphism can be induced directly by $D_{X_{1}}$ without the intercession of $C_{1}^{p}$. However, this follows easily from the commutativity of the diagram below and the fact that the isomorphism of $H^{p}\left(C^{*}\right)$ with $H^{p}\left(C_{1}^{*}\right)$ is given by $\delta_{X}$.

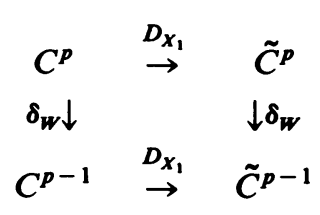

This finishes the lemma.

REMARK. In the above lemma we have not made any claim that the isomorphisms are topological. This is because we have not shown that $W$ has a bounded inverse on either $C^{\infty}(U)$ or $M_{X_{1}}$. Notice, however, that Lemma 9 does not involve $W$ at all except in its proof. We shall obtain the topological properties of the isomorphism later without the aid of Lemma 2. In fact we have not even defined a topology on $M_{X_{1}}$ as yet. This fact we remedy immediately. We give $M_{X_{1}}$ the product topology. Notice that the action of $X_{1}$ on $M_{X_{1}}$ is essentially a left shift so $X_{1}$ acts surjectively and $X_{1}$ is an open mapping. The following is a counterpart to Lemma 6.

LEMma 10. The action of $X_{1}$ on $\Lambda^{p}\left(\mathcal{H}_{0}, M_{X_{1}}\right)$ is surjective and open.

Proof. The action of $X_{1}$ on $f \in \Lambda^{P}\left(\mathcal{F}_{0}, M_{X_{1}}\right)$ is given by

$$
X_{1} \cdot f=\left(X_{1}-X_{1}^{*}\right) f
$$

where $X_{1}^{*}$ is as defined previously (Lemma 6). As a formal power-series

$$
\left(X_{1}-X_{1}^{*}\right)^{-1}=\sum_{n=0}^{\infty} X_{1}^{-(n+1)}\left(X_{1}^{*}\right)^{n}
$$


We let $f_{n}$ be such that

(i) $X_{1}^{(n+1)} f_{n}=\left(X_{1}^{*}\right)^{n} f$,

(ii) $f_{n}=0$ if $\left(X_{1}^{*}\right)^{n} f=0$.

$f_{n}$ is then a finite sequence and it is easily seen that

$$
\left(X_{1}-X_{1}^{*}\right)\left(\sum f_{n}\right)=f \text {. }
$$

This shows surjectivity.

To see openness, let $U$ be a neighborhood of zero in $\Lambda^{p}\left(\mathcal{F}_{0}, M_{X_{1}}\right)$ and consider $\mathcal{V}=\left(X_{1}-X_{1}^{*}\right) \mathcal{Q}$. It suffices to show that $\mathcal{V}$ is a neighborhood of zero. Let $\boldsymbol{n}$ be such that $\left(X_{1}^{*}\right)^{n}=0$ and let $\mathscr{V}_{0}=\bigcap_{k=0}^{n+1}\left(X_{1}\right)^{k} \mathcal{Q}$. Let $\mathfrak{V}_{1}=\cap_{k=0}^{n}\left(X_{1}^{*}\right)^{-k} \mathfrak{V}_{0} \cdot \mathscr{V}_{1}$ is a neighborhood of zero. We claim $\mathcal{V}_{1} \subset \mathcal{V}$. In fact, for $f \in \mathfrak{V}_{1}$, the $f_{n}$ defined above can be chosen in $\mathcal{Q}$ so $f \in\left(X_{1}-X_{1}^{*}\right) \mathcal{U}$. This proves the lemma.

Now we apply the second portion of Lemmas 2 and 3 to $\Lambda^{p}\left(\mathcal{F}_{0}, M_{X_{1}}\right)$ with $\delta=\delta_{X_{1}}$. We come up with the following fascinating conclusion.

LeMMA 11. $H^{p}\left(\mathcal{K}_{0}, C^{\infty}(U)\right)$ is isomorphic with $H^{p}\left(\mathcal{K}_{1}, C^{\infty}\left(U_{1}\right)\right)$.

Proof. $H^{p}\left(\mathcal{H}_{0}, C^{\infty}(U)\right)$ is isomorphic with $H^{P}\left(\mathcal{F}_{0}, M_{X_{1}}\right)$ by the previous lemma. From Lemma $2, H^{p}\left(\mathcal{C}_{0}, M_{X_{1}}\right)$ in turn is isomorphic with $H^{p}\left(\mathcal{G}_{0}, C_{0}^{*}\right)$ where $C_{0}^{*}$ is the intersection of the kernel of $\delta_{X_{1}}$ in $\Lambda^{P}\left(\mathcal{C}_{0}, M_{X_{1}}\right)$ with the kernel of the canonical action of $X_{1}$. A form $f$ is in the kernel of $\delta_{X_{1}}$ iff $f$ is constant on additive cosets of $\operatorname{span}_{\mathbf{C}} X_{1}$ in $\mathcal{K}_{0}$ in each variable. It follows that restriction of such forms to $\mathcal{K}_{1}$ is a vector space isomorphism onto $\Lambda^{P}\left(\mathcal{K}_{1}, M_{X_{1}}\right)$.

Sublemma. Let $f=\left(f_{0}, \ldots\right)$ be a form in $\Lambda^{p}\left(\mathcal{F}_{0}, M_{X_{1}}\right)$. Then $f$ is in the kernel of the canonical action iff

$$
f=\left(f_{0}, X_{1}^{*} f_{0},\left(X_{1}^{*}\right)^{2} f_{0} / 2 !, \ldots,\left(X_{1}^{*}\right)^{n} f_{0} / n !, \ldots\right) .
$$

Proof. $f$ is in the kernel of the canonical action iff $X_{1} f=X_{1}^{*} f$ so

$$
\left(f_{1}, 2 f_{2}, \ldots\right)=\left(X_{1}^{*} f_{0}, X_{1}^{*} f_{1}, \ldots\right) \text {. }
$$

The sublemma follows easily from this.

It is now easily seen that the mapping $C_{0}^{p} \rightarrow \Lambda^{p}\left(\mathcal{H}_{1}, C^{\infty}\left(U_{1}\right)\right)$ given by mapping $f=\left(f_{0}, \ldots\right)$ into the restriction of $f_{0}$ to $\mathcal{K}_{1}$ is a continuous chain isomorphism with continuous inverse. It follows that $H^{p}\left(C_{0}^{*}\right)$ and $H^{p}\left(\mathcal{C}_{1}, C^{\infty}\left(U_{1}\right)\right)$ are isomorphic, even as topological spaces. Our lemma follows.

We may finally finish Subcase II.A. It is clear that $H^{P}\left(\mathcal{H}_{1}, C^{\infty}\left(U_{1}\right)\right)$ and $H^{p}\left(\left(\mathcal{H}_{1}+\operatorname{span}_{\mathbf{C}} Y\right) / Y, C^{\infty}\left(U_{1} / Y\right)\right)$ are identical. It is easily verified that $\mathcal{H}_{1}+$ $\operatorname{span}_{\mathbf{C}} Y / Y$ is a maximal subordinate subalgebra in $\left(\Re_{1}\right)_{c}$ so by induction $H^{p}\left(\mathcal{H}_{1}, C^{\infty}\left(U_{1}\right)\right)$ is zero unless $p$ equals the deficit of $\phi_{\lambda_{1} / Y}$.

LEMMA 12. $\phi_{\lambda_{1}}$ and $\phi_{\lambda_{1} / Y}$ have the same deficit.

Proof. Consider the subspace $\tilde{\mathcal{H}}$ of $\mathcal{K}_{0}$ spanned by $W=Y+i V$ and $X_{1}$. Since $Y$ centralizes $\Re_{1}, \phi_{\lambda}(W, W)=0$. We claim $\phi_{\lambda}\left(W, X_{1}\right) \neq 0$. Of course $\lambda\left(\left[Y, X_{1}\right]\right) \neq$ 0 since $X_{1} \notin \Re_{1}$, while $\lambda\left(\left[Y+i V, X_{1}\right]\right)=0$. Hence $\lambda\left(\left[i V, X_{1}\right]\right)=-\lambda\left(\left[Y, X_{1}\right]\right) \neq 0$. Hence

$$
\phi_{\lambda}\left(X_{1}, Y+i V\right)=-i \lambda\left(\left[X_{1}, Y-i V\right]\right)=-i 2 \lambda\left(\left[X_{1}, Y\right]\right) \neq 0
$$


It follows that $\phi_{\lambda}$ is nondegenerate on $\tilde{\mathcal{H}}$ and there is a canonical basis for $\phi_{\lambda}$ passing through $\tilde{\mathcal{C}}$. The matrix of $\phi_{\lambda}$ on $\tilde{\mathcal{K}}$ is of the form

$$
A=\left(\begin{array}{cc}
a & b \\
\bar{b} & 0
\end{array}\right)
$$

where $a=\phi_{\lambda}\left(X_{1}, X_{1}\right)$ is real and $b=\phi_{\lambda}\left(X_{1}, W\right)$. The characteristic polynomial is $x^{2}-a x-|b|^{2}$. Hence $A$ has one positive and one negative eigenvalue. On the other hand, $W$ is in the radical of the restriction of $\phi_{\lambda}$ to $\mathcal{K}_{1}$. Hence on $\mathcal{K}_{1}, \phi_{\lambda}$ loses one positive eigenvalue and one negative eigenvalue and gains a zero eigenvalue. As the dimension of the subordinate subalgebra also decreases by one, this makes a net change of zero in the deficit. This proves the lemma.

It follows from the lemma that $H^{p}\left(\mathcal{H}, C^{\infty}(U)\right)$ is zero if $p \neq q_{0}$ where $q_{0}$ is the deficit and $H^{q_{0}}\left(\mathcal{H}, C^{\infty}(U)\right)$ is one dimensional. To finish Subcase II we need to show that $H^{q_{0}}\left(\mathcal{H}, C^{\infty}(U)\right)$ is Hausdorff-i.e. the image of $\partial$ is closed in $\Lambda^{q_{0}}\left(\mathcal{H}, C^{\infty}(U)\right)$. This will be the case if the image of $\partial$ is closed in $\Lambda^{q_{0}}\left(\mathcal{H}, M_{X_{1}}\right)$ for the isomorphism of $H^{q_{0}}\left(\mathcal{H}, C^{\infty}(U)\right)$ and $H^{q_{0}}\left(\mathcal{H}, M_{X_{1}}\right)$ is equivalent with the statement that $f$ is a boundary in $\Lambda^{q}\left(\mathcal{H}, C^{\infty}(U)\right)$ iff $f \in D_{X_{1}}^{-1}\left(\partial \Lambda^{q-1}\left(\mathcal{H}, M_{X_{1}}\right)\right)$. The closeness would then follow from the continuity of $D_{X_{1}}$. But from the proof of Lemma $11, H^{q_{0}}\left(\mathcal{H}, M_{X_{1}}\right)$ is topologically isomorphic with $H^{q_{0}}\left(\mathcal{H}_{0}, C^{\infty}\left(U_{1}\right)\right)$. The closure property follows from this. This finishes Subcase II.A.

Subcase II.B. In this case $Y \in \mathscr{H}+\overline{\mathcal{H}}$ but $Y \notin \mathcal{H}_{1}+\overline{\mathcal{K}}_{1}$. Then there is a $V \notin \Re_{1}$ such that $X_{1}=Y+i V \in \mathcal{H}$. We may pick the $X_{0}$ used above equal to $2 c V$ where $c$ is a real constant chosen so that $\lambda([2 c V, Y])=1$. Unfortunately, Subcase II.B now splits into two cases, depending on the sign of $c$.

Assume first that $c>0$.

LEMMA 13. The canonical action of $X_{1}$ on $\Lambda^{p}\left(\mathcal{F}_{0}, C^{\infty}(U)\right)$ is surjective and open. Its kernel is isomorphic with $\Lambda^{p}\left(\mathcal{H}_{0}, C^{\infty}\left(U_{1}\right)\right)$ under the mapping $f \rightarrow f(0)$.

Proof. The openness will follow from the open mapping theorem once surjectivity is shown. We first consider $p=0$. As mappings on $C^{\infty}\left(\mathbf{R}, C^{\infty}\left(U_{1}\right)\right), \partial U\left(X_{0}\right)$ is $d / d s$ and $\partial U(Y)$ is multiplication by is.

$$
\partial U\left(X_{1}\right)=i\left(s+(2 c)^{-1} d / d s\right) .
$$

$g$ is in the image of $\partial U\left(X_{1}\right)$ iff $-2 c i g=(d / d s) f+2 c s f$. A solution of this equation is

$$
f(s)=-2 i c e^{-c s^{2}} \int_{0}^{s} e^{c t^{2}} g(t) d t
$$

provided the $f$ so defined belongs to $C^{\infty}(U)$. To see that $f$ does define an element of $C^{\infty}(U)$, note that it is easily computed by differentiation under the integral that $f$ is valued in $C^{\infty}\left(U_{1}\right)$. The fundamental theorem of calculus combined with differentiation under the integral shows that $f$ is a $C^{\infty}$ mapping of $\mathbf{R}$ into $C^{\infty}\left(U_{1}\right)$. Hence we need only show that $f$ and its derivatives are polynomially bounded. However

$$
(2 c)^{-1}\|f(s)\| \leqslant e^{-c s^{2}} \int_{0}^{s} e^{c t^{2}}\|g(t)\| d t<e^{-c s^{2}} \int_{0}^{s} M e^{c t^{2}}
$$


By l'Hospital's rule this tends to zero as $s \rightarrow \pm \infty$. The polynomial boundedness of $f$ follows similarly using the polynomial boundedness of $g$. The polynomial boundedness of the derivatives of $f$ is proven using the Leibnitz rule for differentiation and l'Hospital's rule as above.

The kernel of $\partial U\left(X_{1}\right)$ is obviously all elements of the form $v e^{-c t^{2}}$ where $v \in$ $C^{\infty}\left(U_{1}\right)$. The isomorphism with $C^{\infty}\left(U_{1}\right)$ is clear.

For $p>0$, the first two statements of the lemma follow as in Lemma 10. To prove the statement about the kernel, suppose $f$ is in the kernel of the canonical action. Then

$$
\partial U\left(X_{1}\right) f-X_{1}^{*} f=0
$$

i.e.

$$
\left(d / d s+2 c s+2 i c X_{1}^{*}\right) f=0 .
$$

To solve this equation we need some notation. If $S$ and $T$ are arbitrary elements of $\Re_{c}$, we let

$$
e^{S}(T)=\sum_{n=0}^{\infty}(\operatorname{ad} S)^{n}(T) / n !
$$

If $\omega$ is a $p$-form on $\mathcal{H}_{0}$, valued in some arbitrary vector space, we set

$$
e^{s} \omega\left(Y_{1}, \ldots, Y_{n}\right)=\omega\left(e^{s}\left(Y_{1}\right), \ldots, e^{s}\left(Y_{n}\right)\right)
$$

whenever $S$ normalizes $\mathcal{H}_{0}$. The relevance of this to our current problem is that

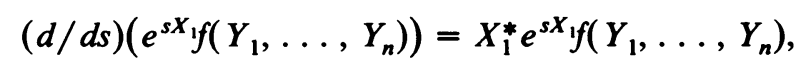

as the reader may easily verify. For $f \in \Lambda^{p}\left(\mathcal{H}_{0}, C(U)\right)$ we set

$$
\tilde{f}(s)=e^{2 s c i X} f(s) \text {. }
$$

Then the equation we wish to solve is equivalent with

$$
(d / d s+2 s c) \tilde{f}(s)=0 .
$$

From the above comments we obtain the solution

$$
f(s)=e^{-c s^{2}} e^{-c i s X} v
$$

where $v$ is a fixed element of $\Lambda^{p}\left(\mathcal{F}_{0}, C^{\infty}\left(U_{1}\right)\right)$. This shows that $f \rightarrow f(0)=v$ is an isomorphism onto $\Lambda^{p}\left(\mathcal{H}_{0}, C^{\infty}\left(U_{1}\right)\right)$ as claimed. This proves the lemma.

We are now in a position to apply Lemmas 2 and 3 with $\delta=\delta_{X_{1}}$ and $X=X_{1}$ in the surjective case. The module $C_{0}^{p}$ is the intersection of the kernel of the canonical action and the kernel of $\delta_{X_{1}}$ so under the isomorphism of Lemma 13, $C_{0}^{p}$ is chain isomorphic with $\Lambda^{p}\left(\mathcal{K}_{1}, C^{\infty}\left(U_{1}\right)\right)$ where $X_{1}$ acts trivially. It follows from Lemmas 2 and 3 that $H^{p}\left(\mathcal{H}_{0}, C^{\infty}(U)\right)$ is topologically isomorphic with $H^{p}\left(\mathcal{G}_{1}, C^{\infty}\left(U_{1}\right)\right)=$ $H^{P}\left(\mathcal{H}_{1}+Y / Y, C^{\infty}\left(U_{1} / Y\right)\right)$. As $\mathcal{H}_{1}+Y$ is maximal subordinate in $\Re_{1} / Y$, we are in a position to apply induction. Our sub-subcase will be done if we show that the deficits of $\phi_{\lambda}$ and $\phi_{\lambda_{1}}$ are the same. Now

$$
\phi_{\lambda}\left(X_{1}, X_{1}\right)=-i \lambda([Y+i V, Y-i V])=c^{-1}>0 .
$$

As $X_{1}$ is orthogonal to the rest of $\mathcal{K}_{1}$, it follows that $\phi_{\lambda_{1}}$ has one fewer positive 
eigenvector than $\phi_{\lambda}$. Since the dimension of $\mathcal{K}_{1}$ is one less than that of $\mathscr{K}_{0}$, the deficit remains constant.

Next suppose $c<0$. In this case we have

LEMMA 14. The canonical action of $X_{1}$ on $\Lambda^{p}\left(\mathcal{H}_{0}, C^{\infty}(U)\right)$ is injective and $X_{1}$ has a continuous inverse defined on its image. $A$ form $f$ is in the image of $X_{1}$ iff

$$
\int_{-\infty}^{\infty} e^{s^{2} c} e^{2 i s c X} f(s) d s=0 .
$$

Proof. As in the proof of Lemma 13, $f$ is in the image of $X_{1}$ iff

$$
-2 i c f=\left(d / d s+2 c s+2 c i X_{1}^{*}\right) g \text {. }
$$

Solving as before we have a solution of the form

$$
g(s)=-2 i c e^{-s^{2} c} e^{-2 i c s X_{1}} \int_{-\infty}^{s} e^{t^{2} c \tilde{f}(t) d t}
$$

where $\tilde{f}(s)=e^{2 i c s X_{1}} f(s)$. Suppose

$$
\int_{-\infty}^{\infty} e^{c t^{2} \tilde{f}(t) d t=0}
$$

We claim that in this case $g$ represents an element of $C^{\infty}\left(U_{1}\right)$. Since $e^{2 i s c X_{1}}$ is polynomial in $s$, one can see that for $g$ to be in $C^{\infty}(U)$ it suffices to show that

$$
h(s)=e^{-s^{2} c} \int_{-\infty}^{s} e^{t^{2} c \tilde{f}(t) d t}
$$

defines an element of $C^{\infty}(U)$.

We claim that $h(s)$ is bounded in $C^{\infty}\left(U_{1}\right)$. To see this let $\phi$ be an element of the continuous dual $C^{-\infty}\left(U_{1}\right)$. From the Banach-Steinhaus theorem, it suffices to show that $\left\langle h(s)\left(Y_{1}, \ldots, Y_{n}\right), \phi\right\rangle$ is bounded for all such $\phi$ and all $Y_{1}, \ldots, Y_{n} \in \mathcal{F}_{0}$. But

$$
\left\langle h(s)\left(Y_{1}, \ldots, Y_{n}\right), \phi\right\rangle=e^{-c s^{2}} \int_{-\infty}^{s} e^{c t^{2}}\left\langle\tilde{f}(t)\left(Y_{1}, \ldots, Y_{n}\right), \phi\right\rangle d s
$$

From l'Hospital's rule, the limit of the left-hand side of the above as $s \rightarrow \infty$ is equal to

$$
\lim _{s \rightarrow \infty}(2 c s)^{-1}\left\langle\tilde{f}(s)\left(Y_{1}, \ldots, Y_{n}\right), \phi\right\rangle=0 .
$$

Note that to apply l'Hospital's rule we need $\int_{-\infty}^{\infty}=0$. It also follows from l'Hospital's rule that the $-\infty$ limit is zero.

Similarly we may prove the polynomial boundedness of $h$. Also, by using the Leibnitz rule for differentiating products and l'Hospital's rule we can show the polynomial boundedness of the derivatives of $h$. This proves the sufficiency part of the lemma.

Conversely, suppose $f$ is in the image of $X_{1}$. Let $g$ be as above. $g$ is the unique solution of $(* *)$ which satisfies $\lim _{s \rightarrow-\infty} g(s)=0$. In order for $g$ to define an element of $\Lambda^{P}\left(\mathcal{F}_{0}, C^{\infty}(U)\right)$ we must have $\lim _{s \rightarrow \infty} h(s)=0$ where

$$
h(s)=(2 i c)^{-1} e^{2 i c s X} \cdot g(s) .
$$

This necessitates the condition on the integral of $\tilde{f}$. 
To see the injectivity of $X_{1}$, it suffices by the proof of Lemma 6 to consider $X_{1}$ on $C^{\infty}(U)$. The kernel of $X_{1}$ is the space of functions of the form $f(s)=e^{-c s^{2}} v$ for $v$ fixed in $C^{\infty}\left(U_{1}\right)$. However, such functions are not square integrable unless $v=0$. Clearly, the integral defining $g$ is a continuous inverse to $X_{1}$ on its image. This proves the lemma.

Now we may apply Lemmas 2 and 3 with $C^{p}=\Lambda^{p}\left(\mathcal{F}_{0}, C^{\infty}(U)\right)$ and $\delta=\delta_{X_{1}}$ so $X$ is given by the canonical action of $X_{1}$. The module $C_{1}^{p-1}$ is $\delta\left(C^{p}\right) / \delta\left(C^{p}\right) \cap$ $X C^{p-1}$. The image of $\Lambda^{p}\left(\mathcal{H}_{0}, C^{\infty}(U)\right)$ under $\delta_{X_{1}}$ is isomorphic with $\Lambda^{p-1}\left(\mathcal{G}_{1}, C^{\infty}(U)\right)$. The above lemma implies that the mapping $D$ of $\delta\left(C^{p}\right)$ into $\Lambda^{p-1}\left(\mathcal{G}_{1}, C^{\infty}\left(U_{1}\right)\right)$ given by

$$
D: f \rightarrow \int_{-\infty}^{\infty} e^{s^{2} c} e^{2 s c i X_{1}} f(s) d s
$$

induces a vector space isomorphism of $C_{1}^{p-1}$ onto $\Lambda^{p-1}\left(\mathcal{H}_{1}, C^{\infty}\left(U_{1}\right)\right)$.

LEMMA 15. $D$ is a chain mapping.

Proof. Let $f \in \Lambda^{p-1}\left(\mathscr{G}_{1}, C^{\infty}(U)\right)$. We define

$$
\tilde{f}(s)=e^{2 i s c X_{1}} f(s)
$$

as before. Note that $2 i s c X_{1}=-s X_{0}+2 i s c Y$. As $Y$ is central in $\Re_{1}$ and $\mathcal{K}_{1} \subset \Re_{1}$,

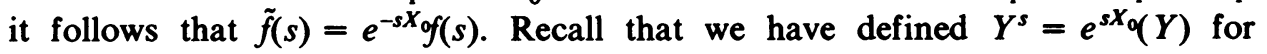
$Y \in \Re_{1}$. The following computation now follows for $Y_{1}, \ldots, Y_{p} \in \mathcal{K}_{1}$.

$$
\begin{aligned}
(\partial f)^{\sim}\left(Y_{1}, \ldots, Y_{p}\right)(s)= & \partial f\left(Y_{1}^{-s}, \ldots, Y_{p}^{-s}\right)(s) \\
= & \sum_{i=1}^{p}(-1)^{i} \partial U\left(Y_{1}^{-s}\right) f\left(Y_{1}^{-s} \cdots Y_{i}^{-s} \cdots Y_{p}^{-s}\right)(s) \\
& -\sum_{i<j}(-1)^{i+j} f\left(\left[X_{i}, Y_{j}\right]^{-s}, Y_{1}^{-s} \cdots Y_{i}^{-s} \cdots Y_{j}^{-s} \cdots Y_{p}^{-s}\right)(s) .
\end{aligned}
$$

Noting that $\partial U\left(Y_{1}^{-s}\right) h(s)=\partial U_{1}\left(Y_{1}\right) h(s)$ for any $h \in C^{\infty}(U)$, we see that $(\partial f)^{\sim}(s)=$ $\partial_{1}(\tilde{f}(s))$ where $\partial_{1}$ refers to the $\Lambda^{p}\left(\mathcal{H}_{1}, C^{\infty}\left(U_{1}\right)\right)$ coboundary operator. Multiplying by $e^{c s^{2}}$ and integrating we get the desired conclusion, thus proving the lemma.

It now follows from Lemma 14 that $C_{1}^{p-1}$ is chain isomorphic with $\Lambda^{p-1}\left(\mathcal{H}_{1}, C^{\infty}\left(U_{1}\right)\right)$. Hence Lemmas 2 and 3 imply the following

Corollary 16. $H^{p}\left(\mathcal{H}_{0}, C^{\infty}(U)\right)$ is topologically isomorphic with $H^{p-1}\left(\mathcal{G}_{1}, C^{\infty}\left(U_{1}\right)\right)$ for $p>0$. If $p=0, H^{p}\left(\mathcal{K}_{0}, C^{\infty}(U)\right)$ is zero.

Again, this will prove our theorem once we show that the deficit $\phi_{\lambda_{1}}$ is one unit less than that of $\phi_{\lambda}$. This is a calculation similar to that done in the $c>0$ case. We shall omit it here. This finishes Subcase II.B.

Now we proceed to the final case

Case III. $Y \notin \mathcal{K}_{0}+\overline{\mathcal{F}}_{0}$. In this case a very fortunate circumstance occurs which aids immensely in computing the cohomology.

LEMMA 17. There is a real element $X_{0}^{\prime} \in \mathscr{H} \cap \mathscr{T} \sim \mathscr{N}_{1}$ such that $\lambda\left(\left[X_{0}^{\prime}, Y\right]\right)=1$. 
Proof. Let $X_{0}$ be as above-i.e. $X_{0} \in \Re \sim \Re_{1}, \lambda\left(\left[X_{0}, Y\right]\right)=1$. Since $\left(\Re_{1}\right)_{c}+$ $\mathcal{H}=\mathcal{T}_{c}$ we may write $X_{0}=Y_{1}+H_{1}$ where $Y_{1} \in\left(\mathcal{R}_{1}\right)_{c}$ and $H_{1} \in \mathcal{H}$. We define a new linear functional $\lambda^{\prime}$ on $\operatorname{span}_{c}\left(\mathcal{F}_{0}+\overline{\mathscr{F}}_{0}+Y+\mathscr{Z}\left(\mathcal{T}_{c}\right)\right)$ by

(i) $\lambda^{\prime}(X)=\lambda\left(\left[Y_{1}, X\right]\right), X \in \operatorname{span}_{\mathbf{C}}\left(Y+\mathcal{H}_{0}+\mathscr{Z}\left(\mathscr{N}_{c}\right)\right)$,

(ii) $\lambda^{\prime}(X)=\lambda^{\prime}(\bar{X})^{-}, X \in \overline{\mathcal{K}}_{0}$.

This is well defined, for if $X \in \mathcal{F}_{0} \cap \overline{\mathcal{F}}_{0}$, then

$$
\lambda\left(\left[Y_{1}, X\right]\right)=\lambda\left(\left[Y_{1}+H_{1}, X\right]\right)=\lambda\left(\left[X_{0}, X\right]\right) .
$$

Hence, since $X_{0}$ is real,

$$
\lambda\left(\left[Y_{1}, \bar{X}\right]\right)=\lambda\left(\left[X_{0}, \bar{X}\right]\right)=\lambda\left(\left[X_{0}, X\right]\right)^{-}=\lambda\left(\left[Y_{1}, X\right]\right)^{-} .
$$

Clearly $\lambda^{\prime}$ satisfies $\lambda^{\prime}(\bar{X})=\lambda^{\prime}(X)^{-}$. Thus $\lambda^{\prime}$ is a real linear functional. We extend $\lambda^{\prime}$ linearly to $\Re$ and then by complex linearity to $\Re_{c}$.

Now, the form $B_{\lambda}$ is nondegenerate on $\mathscr{T} / \mathscr{Z}(\mathcal{K})$ and $\lambda^{\prime}$ is trivial on $\mathscr{Z}(\Re)$. Hence there is a $Y_{2} \in \mathcal{N}$ such that $\lambda^{\prime}(X)=B_{\lambda}\left(Y_{2}, X\right)$. Now for $X \in \mathcal{H}_{0}$

$$
\lambda^{\prime}(X)=B_{\lambda}\left(Y_{1}, X\right)=B_{\lambda}\left(Y_{2}, X\right) \text {. }
$$

Thus $Y_{1}-Y_{2}$ annihilates $\mathcal{H}_{0}$ relative to $B_{\lambda}$. Hence from the maximality of $\mathcal{H}$, $Y_{1}-Y_{2} \in \mathcal{H}$. It follows that in the decomposition $X_{0}=Y_{1}+H_{1}$ we may take $Y_{1}=Y_{2}$. Thus $X_{0}^{\prime}=X_{0}-Y_{1}$ is a real element of $\Re$ which also belongs to $\mathcal{H}$. Furthermore, it follows from $\lambda^{\prime}\left(Y_{2}\right)=0$ that $Y_{1} \in \Re_{1}$ so $\lambda\left(\left[X_{0}^{\prime}, Y\right]\right)=1$ as desired. This proves the lemma.

We, of course, now choose $X_{0}=X_{0}^{\prime}$. As $X_{0}$ acts on $C^{\infty}(U)$ as $d / d s$, it is clear that $\partial U\left(X_{0}\right)$ is injective on $C^{\infty}(U)$ and hence on $\Lambda^{P}\left(\mathcal{F}_{0}, C^{\infty}(U)\right)$ by Lemma 6. As in the proof of Lemma 14 , it is easily seen that the canonical action of $X_{0}$ has a continuous inverse and a form $f$ in $\Lambda^{p}\left(\mathcal{F}_{0}, C^{\infty}(U)\right)$ is in the image of the canonical action of $X_{0}$ iff

$$
\int_{-\infty}^{\infty} e^{-s X_{0 f}(s) d s=0}
$$

It now follows as in Lemma 15 and Corollary 16 that $H^{p}\left(\mathcal{K}_{0}, C^{\infty}(U)\right)$ and $H^{p-1}\left(\mathcal{H}_{1}+Y / Y, C^{\infty}(U / Y)\right)$ are isomorphic for $p>0$. Case III is then completed by computing the deficit of $\phi_{\lambda_{1}}$. We leave the details to the reader.

This finishes the proof of Theorem 1. Q.E.D.

CONCLUDING REMARKS. Having been through the proofs, the reader may wonder at this point about the roles played by the square integrability and the local injectivity. The roles are, in fact, the same. The local injectivity is to preclude the Lie algebra cohomology of $\mathcal{K}_{0}$ from entering the picture. The necessity of this precaution is exemplified by the case where $U$ is one dimensional so $\mathcal{H}=\mathscr{T}_{c}$. Then $C^{\infty}(U)=\mathbf{C}$ and $\mathscr{K}_{0}$ acts trivially. Then $\Lambda^{p}\left(\mathcal{K}_{0}, C^{\infty}(U)\right)$ is just the $p$ th Lie algebra cohomology space of $\mathcal{K}_{0}$. Certainly our Theorem 1 need not be true in this circumstance.

The role of the square integrability is to insure that the representation $U_{1} / Y$ used in the induction scheme is locally injective. It seems plausible that there is a generalization of Theorem 1 which assumes neither square integrability nor local 
injectivity. Such a theorem probably would require a knowledge of the Lie algebra cohomology of the kernel of $U$. Of course, it is only the locally-injective, square-integrable case which is relevant to holomorphic induction.

\section{BIBLIOGRAPHY}

1. J. Camora, Représentations du groupe de Heisenberg dans les espaces de $(0, q)$ formes, Math. Ann. 205 (1973), 89-112.

2. L. Corwin, F. Greenleaf and R. Penney, A general character formula for irreducible projections on $L^{2}$ of a nilmanifold, Math. Ann. 225 (1977), 21-32.

3. G. Hochschild and J. P. Serre, Cohomology of Lie algebras, Ann. of Math. (2) 57 (1953), 591-603.

4. A. Kirillov, Unitary representations of nilpotent Lie groups, Russian Math. Surveys 17 (1962), 53-104.

5. C. Moore and J. Wolf, Square integrable representations of nilpotent groups, Trans. Amer. Math. Soc. 185 (1973), 445-462.

6. H. Moscovici and A. Verona, Harmonically induced representations of nilpotent Lie groups, Invent. Math. 48 (1978), 61-64.

7. H. Moscovici, A vanishing theorem for $L^{2}$-cohomology in the nilpotent case, (Conference on Non-Commutative Harmonic Analysis, Marseille-Luminy, June, 1978), Springer, Berlin and New York.

8. R. Penney, Harmonically induced representations on nilpotent Lie groups and automorphic forms on nilmanifolds, Trans. Amer. Math. Soc. 260 (1980), 123-145.

9. N. S. Poulsen, On $C^{\infty}$-vectors and intertwining bilinear forms for representations of Lie groups, J. Functional Analysis 9 (1972), 87-120.

10. I. Satake, Unitary representations of semi-direct product of Lie groups on $\bar{\partial}$-cohomology spaces, Math. Ann. 190 (1971), 177-202.

Department of Mathematics, PURdue University, West Lafayette, Indiana 47907 